\title{
Author Correction: Recent advances in neurotechnologies with broad potential for neuroscience research
}

Abraham Vázquez-Guardado (D, Yiyuan Yang, Amay J. Bandodkar and John A. Rogers (D)

Correction to: Nature Neuroscience https://doi.org/10.1038/s41593-020-00739-8, published online 16 November 2020.

In the version of this article initially published, errors occurred in the text and Fig. 2 legend.

In the paragraph beginning "Advances in materials science are essential attempts..." the platinum-silicone composites should have been described as " $300 \mu \mathrm{m}$ electrode diameter, $35 \mathrm{~nm}$ thickness, $1.4 \Omega \mathrm{cm}^{2}$ impedance, $57 \mu \mathrm{C} / \mathrm{cm}^{2}$ CICs." In the paragraph beginning "Microand nanofabrication techniques ..." the array of 360 recording sites for fast addressing should have been described as having sampling rates of $\sim 277 \mathrm{~Hz}$. In the paragraph beginning "Another area of progress ..." single- or few-layer sheets of graphene should have been described as having impedances of $1.6 \Omega \mathrm{cm}^{2}$ and $91 \Omega \mathrm{cm}^{2}$, respectively, while structures defined by colloidal sphere lithography and traditional photolithography should have been described as having impedances of $1.63 \Omega \mathrm{cm}^{2}$ and $0.14 \Omega \mu \mathrm{m}^{2}$, respectively.

In the paragraph beginning "Incorporating lenses and imaging ..." the phrase "at high sampling rates (16 Hz) and fine resolution $(\sim 15 \mu \mathrm{m})$ " should have cited ref. 74 .

In the Fig. 2 legend, panel $\mathbf{f}$ should read "Flexible array of 360 gold electrodes $\left(300 \times 300 \mu \mathrm{m}^{2}\right.$, spaced by $\left.500 \mu \mathrm{m}\right)$ supported by a backplane of active matrix electronics on a thin $(25 \mu \mathrm{m})$ polyimide substrate for micro-electrocorticography $\left(10 \times 9 \mathrm{~mm}^{2}\right)$ from the auditory cortex at a density of 400 electrodes $\mathrm{cm}^{-2}$." The references for panels $\mathbf{g}$ and $\mathbf{h}$ were swapped; they should read "g, ref. 34, Springer Nature; h, ref. 29, AAAS."

The errors have been corrected in the PDF and HTML versions of this article.

Published online: 9 February 2021

https://doi.org/10.1038/s41593-021-00813-9

(C) Springer Nature America, Inc. 2021

\section{Author Correction: The landscape of somatic mutation in cerebral cortex of autistic and neurotypical individuals revealed by ultra-deep whole-genome sequencing \\ Rachel E. Rodin, Yanmei Dou, Minseok Kwon, Maxwell A. Sherman D, Alissa M. D'Gama, Ryan N. Doan, Lariza M. Rento Kelly M. Girskis, Craig L. Bohrson, Sonia N. Kim, Ajay Nadig, Lovelace J. Luquette D , Doga C. Gulhan, Brain Somatic Mosaicism Network*, Peter J. Park (D) and Christopher A. Walsh (D)}

Correction to: Nature Neuroscience https://doi.org/10.1038/s41593-020-00765-6, published online 11 January 2021.

In the version of this article initially published, the second sentence of the Methods section "Comparison of mutational signatures between earlier and later mutations" should have read, "To avoid over-fitting, we extracted the two most common clock-like signatures (signature S1 and signature S5), as well as a reactive oxygen species signature (signature S18) from the PCAWG signatures, and deconstructed mutational signatures for the mosaic mutations using the R package deconstructSigs ${ }^{67}$." The error has been corrected in the PDF and HTML versions of this article.

${ }^{*} \mathrm{~A}$ list of authors and their affiliations appears online.

Published online: 22 March 2021

https://doi.org/10.1038/s41593-021-00830-8

(c) The Author(s), under exclusive licence to Springer Nature America, Inc. 2021 